\title{
Summarisation through Discourse Structure
}

\author{
Dan Cristea $^{1,2}$, Oana Postolache ${ }^{1,3}$, and Ionut Pistol ${ }^{1}$ \\ 1 "Al. I. Cuza" University, Iaşi, Romania \\ pic@infoiasi.ro \\ 2 Centro per la Ricerca Scientifica e Tecnologia, \\ Instituto Trentino di Cultura, Trento, Italy \\ cristea@itc.it \\ 3 Computational Linguistics, Saarland University, Saarbrücken, Germany \\ oana@coli.uni-sb.de
}

\begin{abstract}
In this paper we describe a method to obtain summaries focussed on chosen characters of a free text. Summaries are extracted from discourse structures, which resemble rhetorical trees. They are obtained by exploiting cohesion and coherence properties of the text. Evaluation intends to evidence the contribution of each module in the final result.
\end{abstract}

\section{Introduction}

In this paper we describe an approach to discourse parsing and summarisation that exploits cohesion and coherence properties of texts. We built discourse structures that resemble the RST (Rhetorical Structure Theory [1]) trees, although ours are binary and lack relation names. The output of the parsing process is used to obtain excerpt-type summaries focussed on individual characters mentioned in the text. A combined, pipe-line/parallel/incremental, type of processing is employed. The involved modules are POS-tagging, FDG-parsing, clause segmentation of sentences, construction of elementary discourse trees, detection of noun phrases (NPs), anaphora resolution (AR), discourse parsing and summarisation. To master the combinatorial explosion yield by different sources of ambiguity, a beam-search based processing is employed. We present the architecture of a discourse parsing system and discuss the evaluation methodology. The final evaluation is realised by comparing the summaries outputted by the system against those collected from human subjects.

Section 2 presents the overall method and the architecture of the system. Section 3 gives a quick overview on veins theory, which stays at the basis of the focussed summarisation method. Section 4 presents the method of incremental parsing and the module that assembles elementary discourse trees corresponding to sentences. Section 5 describes how the exponential explosion induced by different sources of ambiguity is controlled. In section 6 the corpus, and the evaluation method are presented and section 7 discusses the results and synthesises some conclusions, limitations, and further work. 


\section{The Method}

We call focussed summary on a character/entity X, a coherent excerpt presenting how $\mathrm{X}$ is involved in the story that constitutes the content of the text. Such summaries are of importance in information retrieval tasks from news or scientific papers when mentions of a certain entity are traced in a document. Note that a generic summary of a discourse sometimes will not include a desired character/entity if this entity appears only collaterally in the given discourse. Suppose, for instance, a drugs company interested to track in medical journals or scientific papers all mentions of a certain drug manufactured by them; neither appropriate extraction of the contexts of the drug mentions in the articles, nor generic summaries of the articles can be of help, as the intention is to know how is the drug mentioned within the general topics of the articles.

We describe the architecture of a system that combines a pipe-line style of processing the text with a parallel and an incremental one, with the aim to obtain an RST-like discourse structure that marks the topology and nuclearity, while ignoring the names of the rhetorical relations. Such trees are then used to compute focussed summaries on certain discourse entities. In the process of building discourse trees, we consider properties of the relationship between reference chains and the discourse structure (a manifestation of cohesion) on one hand and, on the other hand,between reference chains and the smoothness of centering transitions (a manifestation of coherence) [2]. Both reference chains and centering transitions are related with veins expressions computed following the veins theory (VT) [3].

First, the text is POS-tagged, then a syntactic parser (FDG) is run over it. Further, the process is split into two flow: one that segments the sentences into elementary discourse units (edus) and then constructs elementary discourse trees (edts) of each sentence, and another one that detects NPs and then runs an AR-engine to detect coreferential relations. Intermediate files in the processing flow are in the XML format. When two processes join, the resulted files are merged into a single representation. An $e d t$ is a discourse tree whose leaf-nodes are the edus of one sentence. Sentence-internal cue-words/phrases trigger the constituency of syntactically edts from each sentence [4], [5]. Since usually, from a given sentence, more than one such tree can be drawn, for each sentence in the original text a set of $e d t \mathrm{~s}$ is obtained. At this point a process that simulates the human power of incremental discourse processing is started. At any moment in the developing process, say after $n$ steps corresponding to the first $n$ sentences, a forest of trees is kept, representing the most promising structures built by combining in all possible ways all edts of all $n$ sentences. Each such tree corresponds to one possible interpretation of the text processed so far. Then, at step $n+1$ of the incremental discourse parsing, the following operations are undertaken: first, all edts corresponding to the next sentence are integrated in all possible ways onto all the trees of the existing forest; then the resulted trees are scored according to four independent criteria, sorted and filtered so that only a fraction of them is retained (again the most promising after $n+1$ steps). From the final 
wave of trees, obtained after the last step, the highly scored is considered to be the discourse structure. Summaries are computed on this tree.

In [6] a general framework to resolve anaphors is proposed. We use this framework to integrate a model of coreference resolution that deals with most types of anaphors. Centering transitions scores are computed after AR is run, therefore after all references are solved. References and transitions, as well as heuristics for the proper development of a discourse tree, contribute with scores to the overall score of a developing discourse tree. These scores are then used to control the beam-search.

\section{Veins Theory and Focussed Summarisation}

Veins theory (VT) $[3,7]$ is used in the described process to guide the incremental tree building and to synthesize summaries. VT makes two claims: emphasizes the close relationship between discourse structure and referentiality, as an expression of text cohesion, and generalizes Centering Theory (CT) [2] to the global discourse, as an expression of text coherence. Moreover, VT adds a view on summarization (consistent with [4]) and naturally reveals how focused summaries can be produced.

The fundamental intuition underlying an integrated account on discourse structure and accessibility in VT is that the RST-specific distinction between nuclei and satellites limits the range of referents to which anaphors can be resolved; in other words, the nucleus-satellite distinction, superimposed over a tree-like structure of discourse, induces a domain of evocative accessibility (dea) for each anaphor. More precisely, for each anaphor $x$ in a discourse unit $u$, VT hypothesizes that $x$ can be resolved by examining discourse entities from a subset of the discourse units that precede $u$. In this way VT reveals a "hidden" structure in the discourse tree, called vein. The notion of vein synthesizes observations on how references interact with the discourse structure represented as an RST tree in which names of relations were ignored (we will call such a simplified representation an RST-like tree). Considering the hierarchical organization given by the tree structure and the principle of compositionality [4], which induces recursively long-distance relations between edus, these observations can be stated as follows [7]:

- a right satellite or a nucleus can refer its left nuclear sibling;

- a right nucleus can refer its left satellite;

- in a combination $n_{1} s_{1} s_{2}$, with $s_{1}$ and $s_{2}$ satellites of the nucleus $n_{1}$, or $n_{1}$ $s_{1} n_{2}$, with $s_{1}$ a satellite of the nucleus $n_{1}$ and $n_{2}$ a right nuclear sibling of $n_{1}, s_{1}$ is not accessible from neither $s_{2}$, nor $n_{2}$;

- a nucleus blocks the reference from a right satellite to a left satellite.

The vein expression of an $e d u u$ is a list of edus of the discourse, including $u$, which is meant to express the sequence of units that are significant to understand $u$ in the context of the whole discourse. 
VT classifies references into three categories, in accordance with the way they align along the veins. An anaphor, belonging to an $e d u u_{2}$, is said to issue a direct reference, if its most linearly recent antecedent belongs to an $e d u u_{1}$ that is included in $u_{2}$ 's vein. Under the same notations, it issues an indirect reference if $u_{1}$ does not belong to $u_{2}$ 's vein, but there is a more distant antecedent, say belonging to an $e d u u_{0}$, and $u_{0}$ is placed on $u_{2}$ 's vein. If the backward-looking reference chain of the anaphor does not intersect the vein of the anaphor's $e d u$, we have an inferential reference.

VT conjunctures on two types of anaphoric processes: evocative (or immediate) and post-evocative (or inferential). The evocative processes are most frequent, are rapid and can be realised by any referential means, including those as fragile as empty pronouns. They make the discourse fluid and increase the text cohesion. The post-evocative anaphorae are less frequent, induce more inferential load on the reader (hearer) and make use of strong referential means (like proper nouns, for instance).

The vein of an $e d u$ also gives a summary of the whole discourse, focused on that particular unit. Now, suppose one discourse entity is traced and a summary focused on that entity is desired. If there is only one $e d u$ in which the entity is mentioned, the vein expression of that $e d u$ gives a very well-focused summary of the entity. A problem appears if the entity is mentioned in more than just one $e d u$. Because there is no a-priory reason to prefer one of the focused summaries obtained in this way to any of the others, it is clear that a combination of the vein expressions of each edu in which the entity is mentioned should be considered. We have tested more methods of building a final summary from the collection of particular summaries. The first method takes the vein expression of the lowest node of the tree that covers all units in which the entity is mentioned. Since the length of a vein expression is proportional to the deepness of the node in the tree structure, this method results in shorter summaries. The second method considers that particular summary (vein expression) which sums most of the mentions of the entity. The third method simply takes the union of all vein expressions of the units that mention the central entity. Finally, the fourth method builds a histogram from all vein expressions of the units mentioning the central entity and selects all units above a certain threshold. The last two methods are not in themselves vein expressions, and therefore are more prone to incoherent summaries than the first two methods, the last one being the most exposed.

\section{Incremental Parsing}

The basic step in an incremental discourse parser is the integration of an elementary tree $(e d t)$, which corresponds to a sentence, in the tree representing the discourse structure of the discourse parsed so far. Out of the two operations applied at each step during an incremental processing, described in [8], we have considered in the present implementation only adjunction. Cue-words and cuephrases are connectives having a signalling function on: the nuclearity of the 
edus they interconnect, the form of the edt they belong to, and the place on the right frontier of the developing tree where an edt is to be adjoined. Subordinate connectives, like just as, although, as long as, whenever, because, etc., link subordinate clauses (satellite structures) onto regent clauses (nuclear structures), while coordinate connectives, like and, or, etc., usually link sibling nuclear structures [9]. There are also frequent cases when connectives miss completely. In conformity also with [10], we have considered that each edt covers exactly the boundaries of one sentence. Different patterns of arguments for cue-words have been manually selected from a corpus. Fig. 1 depicts some cases (the dots suggest the nuclearities of their arguments). There are frequent cases when the same marker has more than one argument pattern.

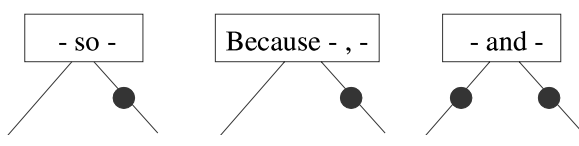

Fig. 1. Arguments patterns of cue-phrases

As constraints to build syntactically correct trees we have used the rules described in [5]. Such constraints configure edts in which inner nodes are labelled with markers and leaf-nodes with edu labels. Each node of the tree is also marked by a nuclearity function with $n$ (for nuclear) or $s$ (for satellite) so that at each level, between the two descendents of an inner node, at least one is marked $n$, and the root of an $e d t$ is always marked $s$. Since the number of inner nodes of a binary tree with $t$ leaf-nodes is $t-1$, for an $e d t$ to be completely determined it needs a number of cue-words, as inner edt nodes, with one less than the number of edus. For such reasons we apply heuristics to add dummy cue-words where missing. Dummy cue-words are empty strings similar to and with both arguments labelled as nuclear (the implicit assumption is that a satellite is announced by a cue-word). The incremental parsing in [5] is deterministic. Heuristics help, at each step, to adjoin the current $e d t$ in that place of the right frontier of the developing tree which maximizes the chances to arrive at a correct final analysis. Instead, our analysis does not go deterministically. At each step, all possible trees resulted from the application of cue-word argument-structure patterns and syntactic constraints are generated and then are adjoined in all possible positions of the right frontier of the developing tree. To control the exponential explosion induced by this luxurious behaviour we implemented a beam-search-like process.

\section{The Beam-Search Control}

Any beam-search-like process depends heavily on a scoring function able to appreciate the relevance of the objects produced at intermediate steps, and which are successively detailed or improved until a final object, supposed to satisfy the goal, is obtained. In this section we explain our scoring criteria. In [11] an empirical evaluation of VT's conjectures is described. Experiments drawn on corpora 
annotated to both discourse structure (RST) and coreference have shown that VT's conjectures are generally correct. The authors of VT report that $87.1 \%$ of all references they found in the investigated corpus are direct, and $8.5 \%$ are indirect. The rest of $4.4 \%$ escape the predictions of VT, some being classified as of a pragmatic type (not needing an antecedent in order to be understood) [3]. In another article [12], the figures reported for references disobeying VT's predictions are greater: $12.3 \%$. However, an important aspect is that exceptions align their frequencies per types with their evoking power, as shown in Table 1:

\begin{tabular}{|c|c|}
\hline type of reference & percent disobeying VT \\
\hline pragmatic & $56.3 \%$ \\
proper nouns & $22.7 \%$ \\
common nouns & $16.0 \%$ \\
pronouns & $5.0 \%$ \\
\hline
\end{tabular}

Table 1. Exceptions from VT's cohesion conjecture, by type of reference

Following [13], the evoking power of each of these types of REs decreases as we move down the list. Pragmatic references are those which refer to entities that can be assumed as part of general knowledge, such as the Senate or our in the phrase our streets. The order in the table suggests that pragmatic references are easily understood without an antecedent while proper nouns and common noun phrases are understood less and less. At the other extreme, pronouns have very poor evoking power: a message emitter employs them only when s/he is certain that the structure of the discourse allows for an easy recuperation of the antecedent in the message receiver's memory. Except for the cases where a pronoun can be understood without an antecedent (as in the example with our in our streets), the use of a pronoun referring an antecedent that is outside the dea should produce an invalid message. Since the detection of pragmatic references requires knowledge that goes beyond the possibilities of our sources, we considered only the last three types of anaphors in Table 1 for the scoring criterion based on references.

To score references in relation with veins we have given the values 2,1 and 0 for the values direct, indirect and outside vein, respectively. Then, to score the anaphor type we have given the values 3, 2 and 1 for the following categories of anaphors: pronoun, common noun and proper noun, respectively. Then we have multiplied these scores for each anaphor, allowing each anaphor to contribute to the general score of the tree with a value between 0 and 6 , with 0 meaning that any of its antecedents are outside the dea of the unit of the anaphor, and 6 in case of a pronoun whose most recent antecedent is on the dea of the unit the anaphor belongs to. This is the $s_{r}$ section of the score (see below).

The second tree-scoring criterion used the coherence conjecture of VT. Following [3], we let each unit to contribute with a score between 0 and 4, depending on the type of centering transition between the current unit and the previous unit in the vein expression, in ascending order of smoothness: no $\mathbf{C}_{b}$, abrupt shift, smooth shift, retaining and continuing [2]. As will be shown below, the score formula is design to keep track of the relationship between references 
and structure. This is the section $s_{c}$ of the score (see below). The overall contribution in the score of a tree coming from VT represents the s1 section of the score formula, and has the following form:

$$
s_{1}=\sum_{u \in D}\left(w_{1} \sum_{x \in R E_{u}} \frac{s_{r}^{x}}{6}+w_{2} \frac{s_{c}^{u}}{4}\right)
$$

where $u$ is an $e d u, D$ represents the whole discourse, $x$ is an anaphor, $R E_{u}$ is the set of the anaphors belonging to unit $u$ which have antecedents outside that unit, $s_{r}^{x}$ is the referential score contributed by the anaphor $x$ and $s_{c}^{u}$ is the centering score contributed by the unit $u$. The two weights $w_{1}$ and $w_{2}$ sum-up to 1 and are iteratively computed to accommodate optimally the score scheme to the expected results ${ }^{1}$.

During the experiments we have noticed a tendency of the parsing trees to be skewed downward and to the right (a tree with this particular shape corresponds to a discourse in which each $e d u$ adds a detail to the preceding one, while a tree completely skewed upward and to the right corresponds roughly to a discourse in which each $e d u$ adds a detail to the initial $e d u$. To balance this tendency we scored better an adjunction of an edt on the upper part of the right frontier of the developing tree than on the lower part. The contribution of this criterion represents the $s_{2}$ section in the score formula, see below.

Section $s_{3}$ of the score formula below is thought to penalize too many nuclear nodes in the final tree. A tree that has only nuclear nodes is a flat structure, but between the two daughters of a node at least one should be nuclear. So, $s_{3}$ is the fraction between the number of satellites and the total number of nodes of the tree.

Finally, the last section of the score - $s_{4}$ - reflects the quality of the edts which are build from sentences. Each edt is compared against the structure returned by the FDG parser (only for English) with respect to the nuclearity of the edus (0.5) and the identity of the sibling node in the structure (0.5) and then we average the sum on the number of edus in the segment.

At each step of the search we have a number $N$ of developing trees and to each of them we adjoin in all possible ways all computed edts. The score of each new developing tree such obtained is calculated as the product $s_{1} * s_{2} * s_{3} * s_{4}$. Then we sort all these trees in the descending order of their scores and we retain for the next step again the first $N$ best rated trees. At the end of the run, the best scored final tree is considered to represent the discourse structure.

\footnotetext{
${ }^{1}$ The careful reader will notice that the weights of this score scheme configure a kind of discourse model, since the number of anaphors per units is not constant, making the two terms of the sum to contribute unequally to the overall score in different discourses. It remains to verify whether the two weights stabilise around some constant values over large corpora, in which case they would indicate the saliance of the two criteria
} 


\section{Corpus and Evaluation}

We have done parallel experiments on both Romanian and English. As a test we have used a fragment summing up 812 words from G. Orwell's novel "1984" in the English version and 863 words in its Romanian equivalent .

We believe that the evaluation of a complex NLP system should follow a procedure that facilitates an easy inventory of the depreciation of performance along the processing chain. This way, the identification of critical points of the system is straightforward and repairing can be focussed towards the points of maximum trouble. In this section we show how we use such a technology in order to evaluate our summarizer for both English and Romanian. The overall processing flow of the system and the points where the "temperature" is measured are depicted in Fig. 2. Early processing phases, as POS-tagging and FDG-parsing are considered included in the input in this scheme. Processing modules are indicated in light grey rectangles, evaluation results in red squares (dark on a black-and-white image), and files - in round rectangles: those which are pure outputs of processing modules - in white, and those influenced in any way by the a gold-standard - in yellow (slightly shadowed). The names of the files indicate their origin, so, for instance np-seg-gold-ar-edt-tree-test is a file that records a gold-standard (gold) of manually annotated noun-phrases (np) and edus (seg), as well as the results (test) of runing the AR-module (ar), the edt-detector module (edt) and the discourse parser module (tree). Also, sumgold and all-test are the two most distant final files, recording respectively the gold-standard of summary and the output of a complete and pure (no human intervention) processing chain.

All initial gold standards, seg-gold, np-gold and np-ar-gold have been created by master students in Computational Linguistics, while the sum-gold file was build with the help of a class of 91 terminal year undergraduate students in Computer Science, during an NLP examination. They received the initial text in which edus were already marked and numbered and were asked to indicate 4 summaries by writing down sequences of discourse unit numbers: a general summary of the whole text of about $20 \%$ reduction rate and three summaries focussed on different characters mentioned in the text (Winston's mother, Winston's sister and the girl with black hair). For each edu of the original text we counted the number of times this $e d u$ was included in any students' summaries. As such, a histogram resulted, with the sequence of $e d u$ numbers on the x-axis and the frequency of mentioning on the y-axis. Then we considered a sliding horizontal threshold on this histogram, and accepted as belonging to the golden summary all units whose corresponding frequencies were above the threshold. During tests we have established the threshold to a number of hits of 20, which resulted in a gold-summary of length 30 edus.

Fig. 2 shows the processing flow and results for the implementation running English texts. In the upper part of the diagram the evaluation points are meant to determine the behaviour of the segment-detector, the NP-detector and the AR-engine, independent of the overall summarization task of the system. In the figure, $\mathrm{P}$ stands for precision, $\mathrm{R}$ for recall and $\mathrm{SR}$ for success rate, conforming 


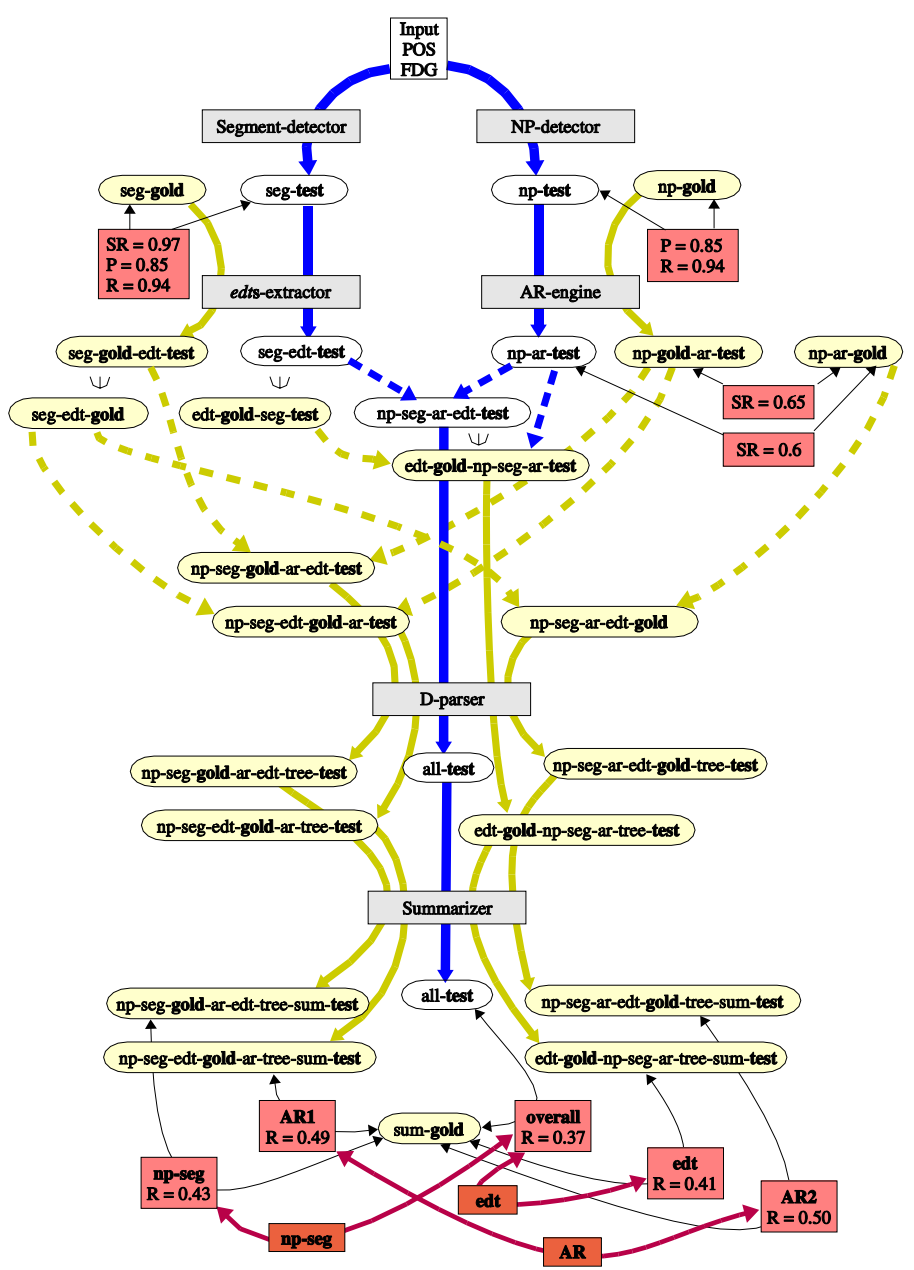

Fig. 2. Processing and evaluation points

to [14]. Precision and recall in the case of segment-detector have been computed in terms of segment borders, while success rate as the number of words correctly assigned to segments (belonging to edus around the same main verb), divided by the total number of words.

As Fig. 2 shows we do not have a gold standard for discourse structure (a file tree-gold is absent). The difficulty to build reliable RST-like annotations over corpora was stressed repeatedly in the literature. To evaluate our trees we used instead summaries, which are much easier to acquire. If summaries extracted automatically, as by-products of a discourse parsing process, resemble those indicated by human subjects, then we should have a high degree of confidence that the structures themselves reflect with enough accuracy the content of the text.

As baseline for our general summaries evaluation we have used the summary produced by MS Word on the same text. As baseline for the three focussed 
summaries we selected all sentences containing the expressions his mother, his sister and girl.

\section{Discussions and Conclusion}

As seen in Fig. 2 the segment-detector behaves satisfactory. A less good precision but very good recall was obtained also for the NP detector. A significant deterioration of the results are expected to occur following the AR-phase since the extreme extravagance of a free text as Orwell's novel and the need to trace at once all types of anaphors made resolution of the coreferring anaphora a very difficult task. Comparing the two SR values (0.65 versus 0.6 ) one can perceive the influence of the NP-detector on the deterioration of the performance of the AR-engine. This behaviour is conformant to the expectations since NPs are the referential expressions that are worked out by the AR-engine. The edts-extractor computed edts as shown in Table 2.

\begin{tabular}{|c|c|c|}
\hline No of edus & $\begin{array}{c}\text { No of sentences } \\
\text { of this length }\end{array}$ & $\begin{array}{c}\text { No of generated } \\
\text { edts per sentence }\end{array}$ \\
\hline $1-3$ & 25 & $1-4$ \\
$4-5$ & 9 & $5-28$ \\
6 & 1 & 42 \\
\hline
\end{tabular}

Table 2. Statistics of the edt-extractor

We tested our discourse parser (D-parser in Fig. 2) over the set of 83 edus which were grouped in 35 sentences in both seg-gold and seg-test.

To master the tree explosion we have used a threshold policy: after each step of the D-parser we kept only the most promising trees whose combined scores range in a threshold of zero under the best score (tie-vote on the maximum). Using this policy, the maximum number of trees generated in any of the 35 steps was 320 .

The implementation was done in Java. To learn the optimum weight values of parameters $w_{1}$ and $w_{2}$ of formula (1) we have run 10 times the whole parser modifying at each step $w_{1}$ by 0.1 (remember that $w_{2}=1-w_{1}$ ). The final results are shown in Fig. 2. For comparison, the MS Word-baseline was rated with a recall of 0.18 . Also, the best student summary was rated 0.59 .

As seen, the results are above the baseline, although the $\mathrm{R}$ values are still low. The interesting thing is that our values are not much smaller than that of the best human-produced summary. Also, the comparison points, as displayed in Fig. 2, validate the expectations: the more gold components we incorporate, the more accurate are the results. We could estimate the impact of the component modules on the summaries by counting the differences between R-values at the edges of the thick red double arrows: NP-detector + segment-detector, as the difference between np-seg and overall values $=0.06$; edts-detector, as the difference between edt and overall values $=0.04$, and AR-engine, as the difference between AR2 and AR1 values $=0,01$. So, it seems that low level processes, as detection of $\mathrm{NPs}$ and segmentation influence more the summarization results that high level 
processes as edt-detection and AR resolution. The results on Romanian are still under development, but we expect to be under the ones for English because of the lack of an FDG parser.

The following aspects will make the subject of further work: retraining of the process with different heuristics, implementation of the substitution operation in incremental discourse parsing, and improvement of the performances of the modules, starting from the NP detector and the sentence segmentation.

\section{Acknowledgements}

Our thanks go to our students who have done the manual anotations and have produced the summaries that helped to draw the final evaluation. Special thanks go to our colleagues from the Laboratory of Computational Linguistics of the University of Wolverhampton who have given us the FDG annotated version of the "1984".

\section{References}

1. Mann, W.C., Thompson, S.A.: Rhetorical structure theory: A theory of text organization. Text 8:3 (1988) 243-281

2. Grosz, B.J., Joshi, A.K., Weinstein, S.: Centering: A framework for modelling the local coherence of discourse. Computational Linguistics (1995)

3. Cristea, D., Ide, N., Romary, L.: Veins theory: A model of global discourse cohesion and coherence. In: Proceedings of COLING/ACL, Montreal/Canada (1998)

4. Marcu, D.: The Theory and Practice of Discourse Parsing and Summarization. The MIT Press (2000)

5. Cristea, D., Postolache, O., Puşcaşu, G., Ghetu, L.: Local and global information exploited in producing summaries. In: Proceedings of the International Symposium on Reference Resolution and Its Applications to Question Answering and Summarisation, Venice/Italy (2003)

6. Cristea, D., Dima, G.E.: An integrating framework for anaphora resolution. Information Science and Technology 4 (2001) 273-291

7. Cristea, D.: The relationship between discourse structure and referentiality in veins theory. In Menzel, W., Vertan, C., eds.: Natural Language Processing between Linguistic Inquiry and System Engineering. Al. I. Cuza University Publishing House (2003)

8. Cristea, D., Webber, B.: Expectations in incremental discourse processing. In: Proceedings of ACL, Madrid/Spain (1997)

9. Knott, A.: A Data-Driven Methodology for Motivating a Set of Coherence Relations. PhD thesis, Department of Artificial Intelligence, University of Edinburgh (1996)

10. Şoricuţ, R., Marcu, D.: Sentence level discourse parsing using syntactic and lexical information. In: Proceedings of HLT-NAACL, Edmonton, Canada (2003)

11. Cristea, D., Ide, N., Marcu, D., Tablan, V.: An empirical investigation of the relation between discourse structure and co-reference. In: Proceedings of COLING, Saarbücken/Germany (2000) 208-214 
12. Ide, N., Cristea, D.: A hierarchical account of referential accessibility. In: Proceedings of ACL, Hong Kong (2000)

13. Gundel, J., Herberg, N., Zacharski, R.: Cognitive status and the form of referring expressions in discourse. Language 69 (1993) 274-307

14. Mitkov, R.: Anaphora Resolution. Longman, London (2002) 